\title{
False memory for associated word lists in individuals and collaborating groups
}

\author{
Ruth H. Maki, Arne Weigold, and Abbigail Arellano \\ Texas Tech University, Lubbock, Texas
}

\begin{abstract}
Using the Deese/Roediger-McDermott (DRM) paradigm, we investigated recall of presented and nonpresented associated words by collaborating groups, nominal groups, and individuals. In Experiment 1, participants recalled individually and then recalled in collaborating groups. Nominal groups made up of individual recall produced more presented and nonpresented associated words than did collaborating groups. Collaborating groups recalled more presented words than did individuals, but not more nonpresented words. In Experiment 2, collaborating groups versus individuals was a between-subjects variable, and everyone made two recall attempts. For recall, the pattern was the same as that in Experiment 1, in that collaborating groups recalled more presented words than did individuals but about the same number of nonpresented words. In a DRM paradigm, collaborating groups were able to produce more presented words than were individuals, without increasing their false recall.
\end{abstract}

When individuals come together, they often attempt to remember events as a group. Generally, groups can recall more information than can individuals (Vollrath, Sheppard, Hinsz, \& Davis, 1989), but the focus of the present experiments was whether they also recall more false information. We used the Deese/Roediger-McDermott (DRM) paradigm (Deese, 1959; Roediger \& McDermott, 1995) to induce false memory in the laboratory. Lists of words associated to nonpresented critical words were presented and recalled by groups or individuals.

Studies of the influence of groups on memory in which the DRM paradigm has been used have taken two general forms. One is the social contagion paradigm, in which a confederate either recalls or recognizes the nonpresented critical words, and the influence of this false memory on individual memory is studied. Such studies have shown that exposure to the false memory of the confederate increases the likelihood that actual participants will also produce false recall (Basden, Reysen, \& Basden, 2002) and recognition (Reysen, 2007) of the nonpresented critical words on individual memory tests. This increase in individual false memory as a result of exposure to the false memory of a confederate occurs both when groups are virtual, with confederates typing their false recall into a computer (Basden et al., 2002), and when confederates are present and verbalize their false recall (e.g., Roediger, Meade, \& Bergman, 2001, using recall of objects in a scene) or false recognition (Reysen, 2007, using DRM lists).

The second phenomenon that has been studied using groups and the DRM paradigm is collaborative inhibition (Weldon \& Bellinger, 1997). Individuals recall first, and all of the words produced by the individuals in a group are considered to be the group's nominal recall. Then the indi- viduals recall as a group, and the group's recall is compared with the nominal recall of the individuals. Nominal groups almost always produce more recall than do individuals (Basden, Basden, Bryner, \& Thomas, 1997; Basden, Basden, \& Henry, 2000). Using the DRM paradigm and virtual groups, Basden, Basden, Thomas, and Souphasith (1998) found that presented words were recalled better by nominal groups than by actual groups, showing collaborative inhibition. However, nominal and actual groups did not differ in the number of nonpresented critical words they recalled.

In the social contagion studies (Reysen, 2007; Roediger et al., 2001) and in Basden and Basden's studies (Basden et al., 1997; Basden et al., 2000; Basden et al., 1998), there was no collaboration, in the sense that individuals did not discuss recall but were exposed to the recall of other group members. In studies of collaborative recall in which groups discuss and agree upon their recall, nominal groups recall more than do collaborating groups, but collaborating groups recall more than do individuals (Finlay, Hitch, \& Meudell, 2000; Weldon \& Bellinger, 1997). Collaborating groups also recall more than do individuals in a situation related to jury deliberation. Pritchard and Keenan (2002) studied individual recall for a videotape of a murder trial before and after group collaboration. They found that deliberation increased accurate recall of peripheral information, but not of central information. In addition, recall following group deliberation contained fewer omission errors than had been made by individuals in their first recall, but the overall number of distortion errors did not change. However, specific distortion errors that were discussed by the group were more likely to be corrected on the second individual recall than were distortion errors that were not discussed.

R. H. Maki, ruth.maki@ttu.edu 
Studies of group recognition have generally shown that collaborating groups produce higher hit rates than do individuals but have shown inconsistent effects for false alarm rates. Clark, Hori, Putnam, and Martin (2000) found that with 2-person collaborating groups, the false alarm rate for the group was higher than the average for the individuals in the group but that the false alarm rate for the group was lower than the average for the individuals with 3-person groups. Rajaram and Pereira-Pasarin (2007) investigated individual recognition of pictures and words following group collaboration or individual restudy of the materials. They found no difference in hit rates but a decrease in false alarm rates following group collaboration, both on an immediate and on a delayed test. Rajaram and Pereira-Pasarin noted that their materials were not designed to produce false memories, but materials that are so designed (such as the DRM materials) result in an increase in false memory following group collaboration. They cited the group studies that did not involve collaboration (Basden et al., 2002; Roediger et al., 2001) as evidence for an increase in false recall. Similarly, Gallo (2006) cited studies that did not involve group collaboration (Basden et al., 2002; Roediger et al., 2001) as evidence for an increase in nonpresented critical word recall in DRM tasks with group recall.

However, there is no evidence that group collaboration increases false recall in the DRM paradigm for groups that actually discuss their collective recall. Pritchard and Keenan (2002) provided some contrary evidence, in that group discussion of specific points reduced memory distortions, but they used a realistic transcript of a trial as their memory material. Whether or not groups actually collaborate seems to be particularly important in determining whether group memory is better or worse than individual memory, particularly for false alarms (Rajaram \& Pereira-Pasarin, 2007).

In the present study, we compared recall in the DRM paradigm for groups that actually collaborated, nominal groups made by pooling individuals' recall, and individuals. We expected that collaborating groups would remember more presented words than would individuals but fewer presented words than would nominal groups. Recall of nonpresented critical words could show a social contagion effect (e.g., Reysen, 2007; Roediger et al., 2001). If one individual falsely recalls a nonpresented critical word, he or she may expose the group to that nonpresented target and influence the group to produce it in recall. In addition, recall by one group member may cue a list that was not accessible by other group members, increasing recall of both presented and nonpresented list words. The alternative view is that groups can eliminate the memory errors of individuals. Pritchard and Keenan (2002) found that individual errors were more likely to be corrected on the second recall if the group had discussed them than if it had not. If collaborating groups can filter errors out of their recall, nonpresented critical words will be less likely to occur in the recall of collaborating groups than in the recall of nominal groups. In Experiment 1, individual versus collaborating group recall was manipulated within subjects; in Experiment 2, individual versus collaborating group recall was manipulated between subjects.

\section{EXPERIMENT 1}

\section{Method}

Participants. Forty introductory psychology students at Texas Tech University volunteered for this experiment and received class credit for their participation. There was a total of 14 groups. Four groups had 2 participants, 8 groups had 3 participants, and 2 groups had 4 participants.

Materials. A prerecorded cassette tape containing 10 lists of 15 associatively related words (Roediger \& McDermott, 1995) was used. Each word in a list was an associate of a nonpresented critical word. The words in each associated list were recorded consecutively at a rate of $2 \mathrm{sec} /$ word, with a 4 -sec pause between lists.

Procedure. The participants were seated at separate tables and were instructed to listen to a cassette tape of words that they would later be asked to recall. The tape presented 10 blocked word lists, each containing 15 words. Following the listening portion, each of the participants was given a blank sheet of paper and was instructed to individually recall as many of the words from the lists as they could, in any order. The participants were given up to $4 \mathrm{~min}$ to complete the free recall, but all reported that they were finished in less time. Recall sheets were collected by the experimenter when the participants indicated that they had finished.

The participants then were instructed to move their chairs together to form a group. The group was instructed to complete a group free recall, with one person writing the words as the group agreed on them. No stipulations regarding the method by which the group conducted the recall were given. Some of the groups took turns, with each individual recalling as much as he or she could before moving on to the next group member. Many of the groups recalled words by list, with each person adding to the list before moving on to the next list. No instructions were given regarding group disagreements. After recall was complete, the participants were debriefed and thanked for their participation. ${ }^{1}$

\section{Results}

We calculated three different measures of recall and confidence. Group was used as the unit of analysis in each case. For individual scores, we found the mean percentages of presented word and nonpresented word recall for each individual and averaged across the members of each group. For the nominal groups, we counted each different word recalled by individual members of the 2- to 4-person groups as a correct or false recall and converted these to percentage of the total number of words. This gave a measure of how many different words had been produced in the individual recall portion by the group members. Finally, we scored group recall by counting the number of presented and nonpresented words recorded by the group in the separate recall that was produced during group collaboration and converted these to percentage of total words.

Figure 1 shows the percentages of presented and nonpresented words recalled by individuals, by nominal groups created from individual recall, and by the groups during the collaboration period. ${ }^{2}$ These percentages were analyzed in a 2 (type of word) $\times 3$ (group condition) repeated measures ANOVA with group as the unit. Overall, a higher percentage of presented words than of nonpresented words was recalled $[M=30.03 \%$ vs. $M=21.76 \% ; F(1,13)=8.91$, $\left.M S_{\mathrm{e}}=161.22, p<.05, \eta_{\mathrm{p}}^{2}=.41\right] .{ }^{3}$ There was a significant main effect of group condition $\left[F(2,26)=48.15, M S_{\mathrm{e}}=\right.$ $\left.68.28, \eta_{\mathrm{p}}^{2}=.79\right]$ and an interaction between type of word and group condition $\left[F(2,26)=13.42, M S_{\mathrm{e}}=36.00, \eta_{\mathrm{p}}^{2}=\right.$ $.51]$. In order to understand the interaction, we compared 


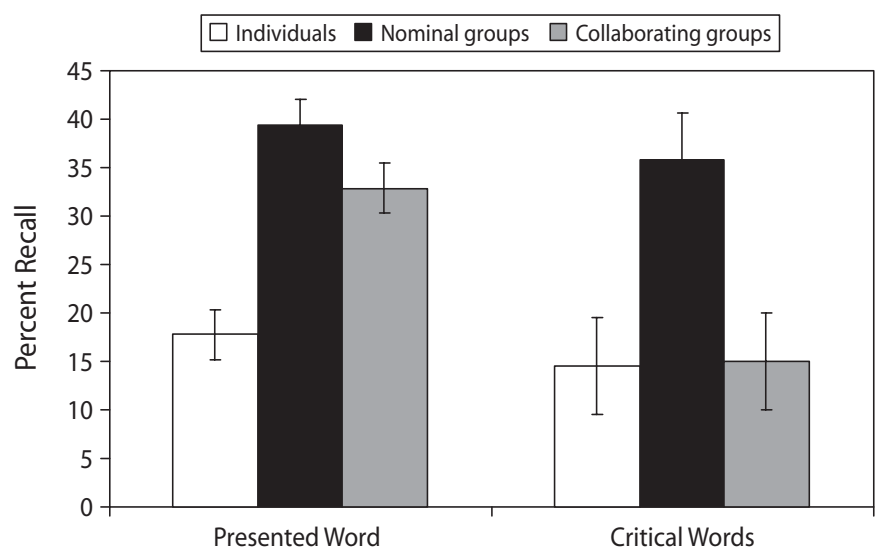

Type of Word

Figure 1. Percentages of recall in Experiment 1 of presented words and nonpresented critical words by individuals, nominal groups, and collaborating groups. Error bars are $95 \%$ confidence intervals, using the pooled variance (group $\times$ participants), computed separately for presented words and nonpresented critical words.

the three group conditions separately for presented words and for critical nonpresented words.

The simple main effect for group with presented words was significant $\left[F(2,26)=76.60, M S_{\mathrm{e}}=22.54, \eta_{\mathrm{p}}^{2}=.86\right]$. Planned pairwise comparisons showed that individuals recalled fewer words $(M=17.79 \%)$ than did actual groups $(M=32.86 \%)\left[F(1,13)=81.88, M S_{\mathrm{e}}=38.84, \eta_{\mathrm{p}}^{2}=.86\right]$. In addition, nominal groups recalled more words $(M=$ $39.45 \%)$ than did actual groups $(M=32.86 \%)[F(1,13)=$ $\left.27.82, M S_{\mathrm{e}}=21.86, \eta_{\mathrm{p}}^{2}=.68\right]$. Thus, we found both facilitation of group over individual recall and collaborative inhibition by collaborating groups, as compared with nominal groups.

Group recall was higher than individual recall because groups accessed more lists and because they recalled more words from accessible lists. Groups recalled at least one word from an average of 8.64 of the 10 lists, whereas individuals recalled at least one word from an average of 6.83 lists. Greater list accessibility for groups was significant $\left[F(1,13)=92.67, M S_{\mathrm{e}}=0.25, \eta_{\mathrm{p}}^{2}=.87\right]$. Groups also recalled a higher percentage of words from accessible lists $(56.4 \%)$ than did individuals $(38.6 \%)[F(1,13)=78.62$, $\left.M S_{\mathrm{e}}=28.36, \eta_{\mathrm{p}}^{2}=.86\right]$.

For nonpresented critical words, the difference among individuals, nominal groups, and collaborating groups shown in Figure 1 was significant $[F(2,26)=25.01$, $\left.M S_{\mathrm{e}}=81.74, \eta_{\mathrm{p}}^{2}=.66\right]$. Individuals recalled about the same number of nonpresented critical words as did collaborating groups $(M=14.57 \%$ vs. $M=15.00 \%)$; the small difference was not significant $(F<1)$. However, nominal groups showed significantly more false recall than did collaborating groups $[35.71 \%$ vs. $15.00 \% ; F(1,13)=$ $\left.23.72, M S_{\mathrm{e}}=253.30, \eta_{\mathrm{p}}^{2}=.65\right]$.

Groups can fail to recall some words that individuals had recalled, and they can also add words that were not recalled by individuals. We were interested in the composition of the words recalled by the collaborating groups. First, we investigated the percentage of presented and nonpresented critical words that were dropped from the nominal group's available words by the collaborating group. Collaborating groups dropped $15.36 \%$ of the nominal groups' recalled presented words and $43.14 \%$ of the nominal groups' recalled nonpresented critical words. This difference was significant $\left[F(1,13)=8.66, M S_{\mathrm{e}}=623.71, \eta_{\mathrm{p}}^{2}=.44\right]$. Second, we calculated the number of words added by the group that were not recalled by any individual in the group. Of the presented words recalled by the groups, $10.57 \%$ had not been recalled by any individual in the group. However, no nonpresented critical words were added by the groups.

\section{Discussion}

Consistent with previous literature, collaborating groups recalled more presented material than did individuals (Pritchard \& Keenan, 2002; Weldon \& Bellinger, 1997). Collaborating groups accessed more lists during their recall than did individuals, and they also recalled more words per list. However, groups did not differ significantly from individuals in recall of nonpresented critical words that were highly associated to presented words. Although social contagion paradigms (Basden et al., 2002; Roediger et al., 2001) in which the false recall of others has increased the false recall of individuals have been cited as evidence for increases in false memory by groups (Gallo, 2006), we found that the results from the social contagion paradigm do not generalize to collaborating groups. In addition, our results contrast with previous results with recognition in which collaborating groups reduced false alarm rates, relative to individuals (Clark et al., 2000, with 3-person groups; Rajaram \& Pereira-Pasarin, 2007). Our results are most similar to those of Pritchard and Keenan (2002), who found that group deliberation increased correct recall while not changing the overall level of incorrect recall, although errors were more likely to be corrected if they were discussed than if they were not discussed. 
In contrast to the results of the comparison of average individual recall and group recall, nominal groups (consisting of all the words recalled by individuals) recalled more presented and nonpresented critical words than did collaborating groups. In considering words that had been recalled individually by group members, groups dropped more nonpresented critical words than presented words. Collaborating groups also added presented words, but not nonpresented critical words. Together, these facts lead to the conclusion that collaborating groups produced fewer false memories than did nominal groups.

This finding contrasts with that of Basden et al. (1998), who compared DRM list recall of nominal and virtual groups who did not collaborate. Similar to our results, their nominal groups recalled more presented words than did actual groups. However, their nominal groups did not recall more nonpresented critical words than did their actual groups. The difference between Basden et al.'s (1998) results and our results probably lies in the fact that our groups collaborated to produce their group recall and this collaboration resulted in the elimination of many nonpresented critical words.

\section{EXPERIMENT 2}

One difficulty in interpreting Experiment 1 is that group recall always followed individual recall. A similar procedure was used by Pritchard and Keenan (2002) in their study of mock trial recall, and they found similar effects - that is, more accurate recall by groups without an increase in false memories. The increase in correct recall could have occurred because recall was repeated. However, this seems unlikely, because Payne, Elie, Blackwell, and Neuschatz (1996) used a DRM paradigm and showed that actual word recall did not change across three tests (with no intervening study) but that false recall increased. Similarly, Burns, Martens, Bertoni, Sweeney, and Lividini (2006) found that recall of list items decreased across three recall attempts but that recall of critical nonpresented words increased significantly.

The pattern that we observed-that is, increases in list word recall but no increase in critical nonpresented word recall - is the reverse of what Payne et al. (1996) and Burns et al. (2006) found. However, it would be useful to show that our group versus individual effects were not simply due to repeated recall by individuals who happened to be in groups for their second recall. Our second experiment included repeated testing for both groups and individuals in a DRM paradigm. Group versus individual recall was a between-subjects variable. We used only 3 - or 4-person groups because Clark et al. (2000) had found that false recognition increased for 2-person groups but decreased for 3 -person groups. ${ }^{4}$

\section{Method}

A total of 71 volunteers from the same pool as that in Experiment 1 participated for partial course credit. Seventeen of the participants were assigned to the individual condition, and 54 participants were assigned to 17 groups of 3-4 individuals. Fourteen of the groups had 3 persons, and 3 of the groups had 4 persons.

The same cassette tape of the blocked DRM lists as that in Experiment 1 was used to present the words. After all the words had been presented, groups and individuals wrote the words that they could recall. The recall sheet was removed, and the participants were asked to recall the words for a second time. ${ }^{5}$ In the individual condition, both recall attempts were made by each individual. The participants in the groups were treated similarly. After hearing the word lists, the groups were asked to select a writer to record the recalled words and to recall as many words as possible as a group. Again, they were not given instructions about how to resolve disagreements. After the recall sheet had been removed, each group was asked to recall a second time.

\section{Results}

We did not form nominal groups using the individuals in the collaborating groups in this experiment because we did not have individual recall in the group condition. Therefore, we compared recall by the collaborating groups with recall by different individuals. Figure 2 shows recall of presented and nonpresented critical words for groups and individuals on each recall attempt. These data were analyzed in a 2 (group condition) $\times 2$ (type of word) $\times 2$ (recall attempt) mixed design ANOVA. First versus second recall attempts did not differ significantly $[24.4 \%$ vs. $23.7 \% ; F(1,32)=$ $\left.1.10, M S_{\mathrm{e}}=16.68\right]$, and recall attempt did not interact with other variables $\left[F \mathrm{~s}(1,32) \leq 1.99, M S_{\mathrm{e}}=16.68\right]$. Therefore, the pattern of recall for presented and nonpresented words did not change across recall attempts.

Percentage of recall was higher for presented words than for nonpresented words $[27.9 \%$ vs. $20.1 \% ; F(1,32)=$ $\left.9.55, M S_{\mathrm{e}}=214.20, \eta_{\mathrm{p}}^{2}=.23\right]$, and groups recalled more than did individuals $[27.1 \%$ vs. $20.9 \% ; F(1,32)=4.32$, $\left.M S_{\mathrm{e}}=303.18, \eta_{\mathrm{p}}^{2}=.12\right]$. More important, there was an interaction between type of word and group condition $\left[F(1,32)=5.55, M S_{\mathrm{e}}=214, \eta_{\mathrm{p}}^{2}=.15\right]$. In order to understand the interaction, we compared the group and individual conditions separately for presented words and for critical nonpresented words.

For presented words, groups recalled significantly more than did individuals $[33.9 \%$ vs. $21.8 \% ; F(1,32)=18.98$, $\left.M S_{\mathrm{e}}=131.53, \eta_{\mathrm{p}}^{2}=.37\right]$. Thus, we again found facilitation of memory for presented words by groups, as compared with individuals. For critical nonpresented words, however, the simple main effect of group condition was not significant $(F<1)$. As can be seen in Figure 2, there was essentially no difference between the levels of false recall for collaborating groups and individuals.

Individuals accessed 7.94 of the 10 lists, and groups accessed 8.65 of the lists. This difference was not significant $\left[F(1,32)=2.75, M S_{\mathrm{e}}=3.08\right]$. The number of lists accessed by the groups is the same as that in Experiment 1 (which was 8.64), but the individuals in this experiment accessed more lists than did those in Experiment 1 (7.94 vs. 6.83). Given that individuals were treated identically in the two experiments, this different pattern across experiments must be due to sampling error. However, as in Experiment 1 , groups recalled more words per accessed list than did individuals $[60.6 \%$ vs. $42.1 \% ; F(1,32)=9.31$, $\left.M S_{\mathrm{e}}=628.60, \eta_{\mathrm{p}}^{2}=.23\right]$.

\section{Discussion}

Experiment 2 replicated Experiment 1 with respect to the DRM results. Groups recalled more presented words than did individuals, but groups did not show increased false 


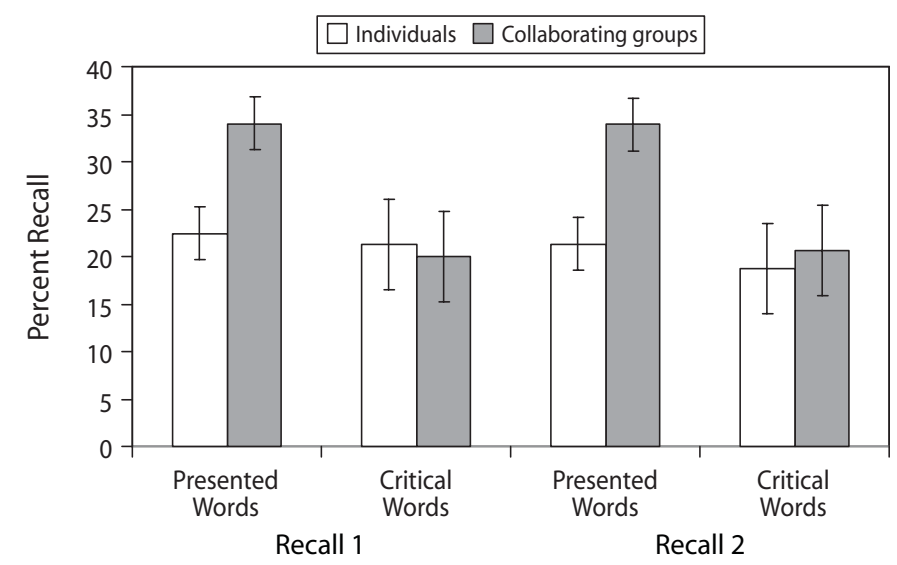

Figure 2. Percentages of recall in Experiment 2 of presented words and nonpresented critical words on the first and second recall attempts by individuals and collaborating groups. Error bars are $\mathbf{9 5 \%}$ confidence intervals, using the pooled variance (participants/group), computed separately for presented words and nonpresented critical words.

recall of nonpresented critical words. In contrast to Burns et al.'s (2006) finding, we did not find significant changes from the first to the second recall attempt in the individuals' recall; neither did we find changes in the groups' recall. Possible reasons for this discrepancy are that our DRM lists ( 15 words per list) were longer than those in Burns et al. ( 5 words per list in Experiment 1 and 10 words per list in Experiment 2) and Burns et al. instructed their participants to be reasonably certain that each word had been presented before writing it down, which we did not include in our instructions. Whatever the reason for this discrepancy in repeated individual recall, we found consistent group versus individual effects across both recall attempts. In this experiment, groups and individuals did not differ significantly in how many lists they accessed, but groups recalled more words per accessed list than did individuals.

\section{GENERAL DISCUSSION}

In two experiments, collaborating groups had higher recall of presented words than did individuals but did not increase their false recall with DRM lists that were designed to produce false recall. In Experiment 2, this effect was not dependent on whether groups were recalling for the first or the second time, so the results of Experiment 1 were not due to the fact that groups were formed only for the second recall attempt. Collaborating groups accessed more words per list in both experiments than did individuals, so the increase in group over individual recall was more than an effect of the cuing of lists that had been missing in individual recall.

These results show that collaborating groups are not influenced by the false recall of individuals in the same way as in social contagion studies (Reysen, 2007; Roediger et al., 2001). In those studies, individuals are exposed to the false recall of others, and they incorporate this false recall into their individual recall. Basden and colleagues have found similar results in studies in which individuals took turns giv- ing public recall responses as part of perceived groups (Basden et al., 1997; Basden et al., 2000; Basden et al., 2002). Collaboration served to reduce the potential memory errors of the groups that were contained in their nominal recall.

Both Gallo (2006) and Rajaram and Pereira-Pasarin (2007) suggested that groups should produce more false memories than individuals do in a paradigm designed to produce incorrect recall, such as the DRM paradigm. However, other evidence suggests that collaborating groups (as opposed to groups recalling in a turn-taking procedure) might produce fewer false memories than do individuals. Rajaram and Pereira-Pasarin found that collaborating groups reduced their false alarm rates in recognition, relative to individuals working alone. Clark et al. (2000) also found lower false alarm rates in recognition with collaborating groups, but only for 3-person, and not for 2-person, groups. Pritchard and Keenan (2002) found that memory distortions of a video-taped mock trial were corrected when participants discussed the specific errors in groups, although the overall number of memory distortions did not differ from before to after group deliberation. The fact that our collaborating groups produced less false recall than did the nominal groups may provide evidence for a similar correction process in the DRM paradigm. However, we did not find that groups produced fewer nonpresented list items than did individuals, just that they produced the same number of false memories while increasing their correct recall. In addition, we cannot be sure that the groups actually discussed the critical lures that had been recalled by a group member but were dropped in group recall.

Collaborating groups did not add nonpresented words that individuals had not recalled, but they did add some presented words. They also reduced the number of critical nonpresented words that they output, relative to the number that was accessible to the groups' individuals. Burns et al. (2006) concluded that critical nonpresented words receive item-specific conceptual processing during learning when they are activated by associated list items. 
This mechanism increases the probability of nonpresented word recall. They also hypothesized that a lack of perceptual features works in parallel to filter the recall of the nonpresented words. Collaborating groups may be more sensitive to the absence of perceptual features of nonpresented words than are individuals, allowing the group to filter these words from their recall.

However, the actual mechanism by which collaborating groups eliminated the false recall that occurred among their individual members is unclear. Pritchard and Keenan (2002) found that individuals' memory distortions were corrected only when the group discussed the errors. Discussion and elimination of false recall may be the reason why our collaborating groups gave fewer falsely recalled words than did the nominal groups. The present results show the need for future researchers to record group interactions and to code which specific items are discussed and eliminated. This should help researchers understand when groups increase false recall, as in social contagion studies, and when they are able to decrease the false recall of individuals, as in Pritchard and Keenan's mock jury study and in our Experiment 1 when nominal groups were compared with collaborating groups.

The present results also suggest that it is important to explain the nature of the group when researchers describe the effects of groups on memory. When the role of the group is to expose individuals to the false recall of others, as in the social contagion paradigm (Roediger et al., 2001) or in virtual groups in which recall is done by turn taking (Basden et al., 2000), more false recall will be produced in individual recall following exposure to others' recall. However, when the group actually collaborates, false alarms may be lower in recognition (Clark et al., 2000; Rajaram \& Pereira-Pasarin, 2007), and memory errors about realistic stimuli may be reduced (Pritchard \& Keenan, 2002). The present experiments now allow us to state what occurs with DRM materials that are designed to produce false memories. Collaborating groups can increase their levels of recall of actually presented words, while not increasing their output of false memories.

\section{AUTHOR NOTE}

Experiment 1 was conducted as an Undergraduate Honors Project by A.A. She is now at Indiana University-Purdue University Indianapolis. Correspondence concerning this article should be addressed to R. H. Maki, Department of Psychology, Texas Tech University, Lubbock, TX 79409-2051 (e-mail: ruth.maki@ttu.edu).

\section{REFERENCES}

Basden, B. H., Basden, D. R., Bryner, S., \& Thomas, R. L., III (1997). A comparison of group and individual remembering: Does collaboration disrupt retrieval strategies? Journal of Experimental Psychology: Learning, Memory, \& Cognition, 23, 1176-1189.

Basden, B. H., Basden, D. R., \& Henry, S. (2000). Costs and benefits of collaborative remembering. Applied Cognitive Psychology, 14, 497-507.

Basden, B. H., Basden, D. R., Thomas, R. L., III, \& Souphasith, S. (1998). Memory distortion in group recall. Current Psychology, 16, 225-246.
Basden, B. H., Reysen, M. B., \& Basden, D. R. (2002). Transmitting false memories in social groups. American Journal of Psychology, 115, 211-231.

Burns, D. J., Martens, N. J., Bertoni, A. A., Sweeney, E. J., \& LiviDINI, M. D. (2006). An item gains and losses analysis of false memories suggests critical items receive more item-specific processing than list items. Journal of Experimental Psychology: Learning, Memory, \& Cognition, 32, 277-289.

Clark, S. E., Hori, A., Putnam, A., \& Martin, T. P. (2000). Group collaboration in recognition memory. Journal of Experimental Psychology: Learning, Memory, \& Cognition, 26, 1578-1588.

DEESE, J. (1959). On the prediction of occurrence of particular verbal intrusions in immediate recall. Journal of Experimental Psychology, 58, $17-22$.

Finlay, F., Hitch, G. J., \& Meudell, P. R. (2000). Mutual inhibition in collaborative recall: Evidence for a retrieval-based account. Journal of Experimental Psychology: Learning, Memory, \& Cognition, 26, 1556-1567.

GaLlo, D. A. (2006). Associative illusions of memory: False memory research in DRM and related tasks. New York: Psychology Press.

Loftus, G. R., \& MAsson, M. E. J. (1994). Using confidence intervals in within-subject designs. Psychonomic Bulletin \& Review, 1, 476-490.

Payne, D. G., Elie, C. J., Blackwell, J. M., \& Neuschatz, J. S. (1996). Memory illusions: Recalling, recollecting, and recognizing events that never occurred. Journal of Memory \& Language, 35, 261-285.

Pritchard, M. E., \& KeEnan, J. M. (2002). Does jury deliberation really improve jurors' memories? Applied Cognitive Psychology, 16, 589-601.

Rajaram, S., \& Pereira-Pasarin, L. P. (2007). Collaboration can improve individual recognition memory: Evidence from immediate and delayed tests. Psychonomic Bulletin \& Review, 14, 95-100.

REYSEN, M. B. (2007). The effects of social pressure on false memories. Memory \& Cognition, 35, 59-65.

Roediger, H. L., III, \& McDermott, K. B. (1995). Creating false memories: Remembering words not presented in lists. Journal of Experimental Psychology: Learning, Memory, \& Cognition, 21, 803-814.

Roediger, H. L., III, Meade, M. L., \& Bergman, E. T. (2001). Social contagion of memory. Psychonomic Bulletin \& Review, 8, 365-371.

Vollrath, D. A., Sheppard, B. H., Hinsz, V. B., \& Davis, J. H. (1989). Memory performance by decision-making groups and individuals. Organizational Behavior \& Human Decision Processes, 43, 289-300.

Weldon, M. S., \& Bellinger, K. D. (1997). Collective memory: Collaborative and individual processes in remembering. Journal of Experimental Psychology: Learning, Memory, \& Cognition, 23, 1160-1175.

\section{NOTES}

1. After individuals and groups completed their recall, they were also asked to rate their confidence in each recalled word. The majority of the ratings ( $86 \%$ of the individual ratings and $93 \%$ of the group ratings) were at maximum confidence. Because there were ceiling effects in the confidence data, they will not be reported.

2 . Error bars on the figures are $95 \%$ confidence intervals. The pooled variance associated with the error term that is most appropriate for the comparisons discussed in the text was used to find the standard error of the mean, as has been suggested by Loftus and Masson (1994). For recall, confidence intervals were determined separately for presented and nonpresented critical words because simple main effects analyses were conducted for each type of word.

3. The significance level used throughout this article is $p<.05$.

4. In Experiment 1, however, reanalyzing the data without the four 2-person groups did not change the pattern of significant results.

5 . We again asked the participants to make confidence judgments for each recalled word. However, as in Experiment 1, there were ceiling effects in the confidence judgments, so we will not report them.

(Manuscript received May 29, 2007; revision accepted for publication October 22, 2007.) 